\title{
Identification of multi-target effects of Huaier aqueous extract via microarray profiling in triple-negative breast cancer cells
}

\author{
XIANGNAN KONG ${ }^{1}$, XIA DING $^{2}$ and QIFENG YANG ${ }^{1,3}$ \\ Departments of ${ }^{1}$ Breast Surgery, ${ }^{2}$ Oncology, Qilu Hospital, Shandong University; \\ ${ }^{3}$ Pathology Tissue Bank, Qilu Hospital, Shandong University, Jinan, Shandong 250012, P.R. China
}

Received January 9, 2015; Accepted February 16, 2015

DOI: 10.3892/ijo.2015.2932

\begin{abstract}
Breast cancer is one of the most common malignant tumors in the world. Long-term maintenance treatment is important for breast cancer. However, effective maintenance treatment is lacking for triple-negative breast cancer (TNBC). Traditional Chinese medicine (TCM) has shown its potential anticancer roles as an effective maintenance treatment for TNBC. However its mechanisms remained unclear. In this study, we detected the differentially expressed genes (DEGs) after treatment with Huaier aqueous extract by using microarray profiling in MDA-MB-231 cells. Gene Ontology (GO) analysis, Kyoto Encyclopedia of Genes and Genomes (KEGG) pathway analysis and gene-gene interaction network were conducted to confirm the altered biological functions induced by Huaier extract. Screening of DEGs gave 387 genes (226 upregulated and 161 downregulated) in MDA-MB-231 cells which were regulated significantly by Huaier extract. GO and KEGG pathway analysis suggested that a number of functions were affected by Huaier, including proliferation, apoptosis, migration, and angiogenesis. Gene-gene interaction network showed the detailed molecular signal-net. Based on microarray data, we studied several functions of Huaier extract and in return verified the results of microarray profiling. This study had important guidance roles and indicated new research directions.
\end{abstract}

\section{Introduction}

Breast cancer is one of the most common malignant tumors around the world. One in 8 women will develop breast cancer in her lifetime in the United States (1). In China, breast cancer

Correspondence to: Professor Qifeng Yang, Department of Breast Surgery, Qilu Hospital, Shandong University, Pathology Tissue Bank, Qilu Hospital, Shandong University, Wenhua Xi Road No. 107, Jinan, Shandong 250012, P.R. China

E-mail: qifengy@gmail.com

Key words: Huaier aqueous extract, microarray profiling, triplenegative breast cancer, differentially expressed genes, Gene Ontology analysis, Kyoto Encyclopedia of Genes and Genomes pathway analysis, gene-gene interaction network is also one of the most common cancers in females. The incidence of breast cancer has increased annually (2). With the help of early diagnosis and multimodality therapy, death rate continues to decline for breast cancer (1-3).

The treatment strategy of breast cancer includes surgery, chemotherapy, radiotherapy, endocrine therapy, target biotherapy and others. However, a considerable number of breast cancer patients inevitably suffer from relapse or metastasis and die from this disease. Long-term maintenance treatment is vital for breast cancer therapy. Endocrine therapy is the important maintenance treatment for estrogenreceptor (ER) positive breast cancer. It can significantly improve the prognosis of premenopausal and postmenopausal patients (4-8). However, for triple-negative breast cancer (TNBC), there is scarce effective maintenance treatment and the prognosis is still poor. Thus new strategies are necessary for the maintenance treatment of TNBC. Traditional Chinese medicine (TCM) has shown its potential anticancer roles. Many agents extracted from TCM have been discovered (9), such as alkaloids $(10)$, flavonoids, polyphenols $(11,12)$ and terpenoids (13), although some of the mechanisms of action have not been elucidated.

Trametes robiniophila murr (Huaier) is a kind of officinal fungi in China. It has been applied in TCM for approximately 1600 years (14) and used as a complementary maintenance treatment of breast cancer in recent decades. Huaier extract consists of many kinds of ingredients. It was confirmed that the most effective ingredient of Huaier extract was proteoglycan, containing $41.53 \%$ polysaccharides, $12.93 \%$ amino acids and $8.72 \%$ water. However, the inhibitory effect of proteoglycan was still less effective than the Huaier extract $(15,16)$. Thus, it is reasonable to speculate that the effects of the Huaier extract may be due to the additive or synergistic effect of different fractions. The antitumor effects of Huaier extract displayed various biological activities, however, the detailed mechanisms of Huaier extract are not clear.

In order to identify the multi-target effects of Huaier as an effective maintenance treatment for TNBC, we detected the differentially expressed genes (DEGs) and altered biological functions of MDA-MB-231 cells after the treatment of Huaier aqueous extract by using microarray profiling in this study. The results of microarray assays indicated that Huaier indeed had various activities in anti-breast cancer treatment and has huge potential for maintenance treatment of TNBC. Under the 
guidance of this study, we have investigated Huaier from many different aspects and have revealed its multiple functions. Therefore, this study provided very important guiding roles for researchers.

\section{Materials and methods}

Cell culture. MDA-MB-231 cells was obtained from American Type Culture Collection (ATCC) (Rockefeller, MD, USA), and routinely cultured in Dulbecco's modified Eagle's medium (DMEM) (Gibco, Rockville, MD, USA) supplemented with $10 \%$ fetal bovine serum (FBS) (Clark Bioscience, Seabrook, MD, USA), $100 \mathrm{U} / \mathrm{ml}$ penicillin and $100 \mu \mathrm{g} / \mathrm{ml}$ streptomycin under the conditions of $5 \% \mathrm{CO}_{2}$ at $37^{\circ} \mathrm{C}$.

Preparation of Huaier aqueous extract. Electuary ointment of Huaier was a kind gift from Gaitianli Medicine Co. Ltd (Jiangsu, China). One gram of the electuary ointment was dissolved in $10 \mathrm{ml}$ of complete DMEM medium and was sterilized with $0.22 \mu \mathrm{m}$ filter to obtain the $100 \mathrm{mg} / \mathrm{ml} \mathrm{stock}$ solution for long storage at $-20^{\circ} \mathrm{C}$.

MTT assay. The MDA-MB-231 cells $\left(1.5 \times 10^{3}\right.$ cells/well $)$ were cultured in 96 -well plates in $5 \% \mathrm{CO}_{2}$ at $37^{\circ} \mathrm{C}$ in complete medium. After incubation overnight, the medium was replaced with different concentrated solutions and incubated for 24, 48 or $72 \mathrm{~h}$ individually. Afterwards, $20 \mu \mathrm{l}$ of 3-(4, 5-dimethylthiazol-2-yl)-2, 5-diphenyltetrazolium bromide (MTT, $5 \mathrm{mg} / \mathrm{ml}$ in PBS) (Sigma-Aldrich, St. Louis, MO, USA) was added to each well and the cells were incubated for another $4 \mathrm{~h}$ at $37^{\circ} \mathrm{C}$. Then the supernatants were carefully aspirated and $100 \mu \mathrm{l}$ of dimethyl sulfoxide (DMSO) (Sigma-Aldrich) was added. The absorbance values were read by Microplate Reader (Bio-Rad, Hercules, CA, USA) at $570 \mathrm{~nm}$.

Cell treatment for microarray profiling. MDA-MB-231 cells suspended at $1 \times 10^{5} / \mathrm{ml}$ density in DMEM with $10 \%$ FBS were incubated in $25 \mathrm{~cm}^{2}$ cell culture flask. The next day the medium was replaced with new medium with and without Huaier aqueous extract $(8 \mathrm{mg} / \mathrm{ml})$ at $37^{\circ} \mathrm{C}$ for $72 \mathrm{~h}$.

HTA 2.0 transcriptome microarray assay. Total RNA was isolated with TRIzol (Invitrogen, Carlsbad, CA, USA) from cells after $72 \mathrm{~h}$ treatment of Huaier aqueous extract $(8 \mathrm{mg} / \mathrm{ml})$. RNAs from three donors with equal amount were pooled together for each sample. Biotinylated cDNA were prepared from $250 \mathrm{ng}$ total RNA according to the Affymetrix standard protocol by using Ambion ${ }^{\circledR}$ WT Expression kit. cDNA were hybridized in Hybridization Oven 645 for $16 \mathrm{~h}$ at $45^{\circ} \mathrm{C}$ on GeneChip Human Transcriptome Array 2.0. GeneChips were scanned by using Affymetrix ${ }^{\circledR}$ GeneChip Command Console (AGCC). The data were analyzed with Robust Multichip Analysis (RMA) algorithm using Affymetrix default analysis settings and global scaling as normalization method. Fold change was used to identify differentially expressed genes (DEGs).

GO analysis. GO analysis was applied to analyze the main function of the differential expression genes according to the Gene Ontology which is the key functional classification of
NCBI, which can organize genes into hierarchical categories and uncover the gene regulatory network on the basis of biological process and molecular function $(17,18)$.

Specifically, two-sided Fisher's exact test and $\chi^{2}$ test were used to classify the GO category, and the false discovery rate (FDR) (19) was calculated to correct the p-value, the smaller the FDR, the small the error in judging the p-value. The FDR was defined as,

$$
F D R=1-\frac{N_{k}}{T}
$$

where $N_{k}$ refers to the number of Fisher's test p-values less than test p-values. We computed p-values for the GOs of all the differential genes. Enrichment provides a measure of the significance of the function: as the enrichment increases, the corresponding function is more specific, which helps us to find those GOs with more concrete function description in the experiment. Within the significant category, the enrichment Re was given by:

$$
\operatorname{Re}=\left(n_{f} / n\right) /\left(N_{f} / N\right)
$$

where ' $n_{f}$ ' is the number of flagged genes within the particular category, ' $n$ ' is the total number of genes within the same category, ' $N{ }_{f}$ ' is the number of flagged genes in the entire microarray, and ' $N$ ' is the total number of genes in the microarray (20).

Pathway analysis. Pathway analysis was used to find the significant pathway of the differential genes according to KEGG, Biocarta and Reatome. The Fisher's exact test and $\chi^{2}$ test were employed to select the significant pathway, and the threshold of significance was defined by P-value and FDR. The enrichment Re was calculated like the equation above (21-23).

Gene-gene Interaction Network. Gene-gene interaction network was constructed based on the data of differentially expressed genes. Using java that allows users to build and analyze molecular networks, network maps were constructed. For instance, if there is confirmative evidence that two genes interact with each other, an interaction edge is assigned between the two genes. The considered evidence is the source of the interaction database from KEGG. Networks are stored and presented as graphs, where nodes are mainly genes (protein and compound) and edges represent relation types between the nodes, e.g. activation or phosphorylation. The graph nature of Networks raised our interest to investigate them with powerful tools implemented in R.

To investigate the global network, we computationally identified the most important nodes. To this end we turned to the connectivity (also known as degree) defined as the sum of connection strengths with the other network genes:

$$
\mathrm{K}_{i}=\sum_{u \neq i} a_{u i}
$$

In gene networks, the connectivity measures how correlated a gene is with all other network genes. For a gene in the network, the number of source genes of a gene is called the indegree of the gene, and the number of target genes of a gene is its outdegree. The character of genes is described by betweenness centrality measures reflecting the importance of a node 
Table I. Top 5 up- and down-regulated genes in Huaier-treatment cells compared with control cells in MDA-MB-231 cells.

\begin{tabular}{lcl}
\hline Gene symbol & Ratio of Huaier/Control & \multicolumn{1}{c}{ Description } \\
\hline MMP3 & 13.0 & Matrix metallopeptidase 3 (stromelysin 1, progelatinase) \\
TCP11L2 & 7.3 & t-complex 11 (mouse)-like 2 \\
IL24 & 6.9 & Interleukin 24 \\
CLEC2B & 4.8 & C-type lectin domain family 2, member B \\
LIPH & 4.7 & Lipase, member H \\
MPP4 & 0.3 & Membrane protein, palmitoylated 4 (MAGUK p55 subfamily member 4) \\
HIST1H2BM & 0.3 & Histone cluster 1, H2bm \\
ID1 & 0.3 & Inhibitor of DNA binding 1, dominant negative helix-loop-helix protein \\
F3 & 0.4 & Coagulation factor III (thromboplastin, tissue factor) \\
SNORD14D & 0.4 & Small nucleolar RNA, C/D box 14D \\
\hline
\end{tabular}

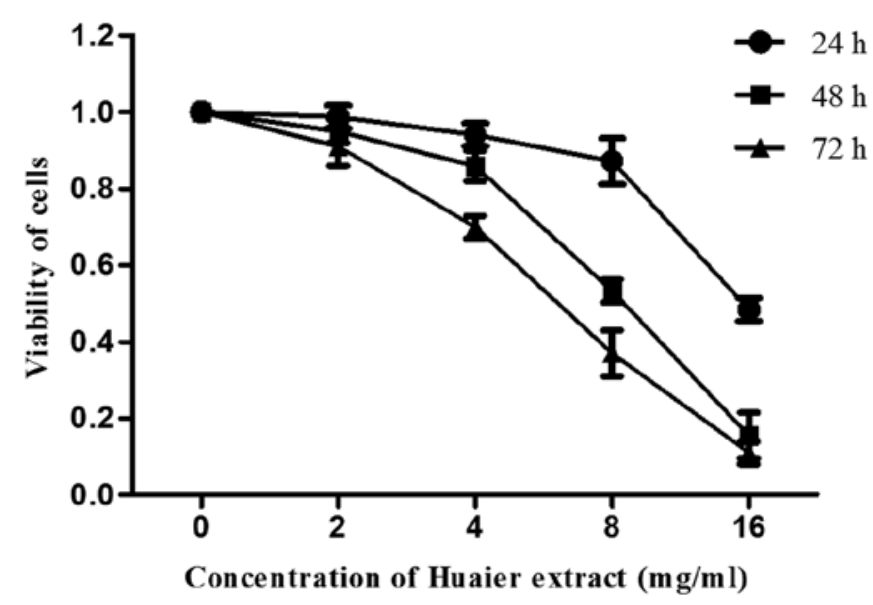

Figure 1 . The inhibitory effect of Huaier on cell viability measured by MTT assay. MDA-MB-231 cells were treated with Huaier for 24, 48 and $72 \mathrm{~h}$. The experiments were performed in triplicate and data presented as the mean \pm SD of three separate experiments.

in a graph relative to other nodes. For a graph $\mathrm{G}:(\mathrm{V}, \mathrm{E})$ with $\mathrm{n}$ vertices, the relative betweenness centrality $C_{B}^{\prime}(v)$ is defined by:

$$
C_{B}^{\prime}(V)=\frac{2}{n^{2}-3 n+2} \sum_{\substack{s \neq v \neq t \in V \\ s \neq t}} \frac{\sigma_{s t}(V)}{\sigma_{s t}}
$$

where $\sigma_{s t}$ is the number of shortest paths from s to t, and $\sigma_{s t}(v)$ is the number of shortest paths from $s$ to that pass through a vertex v (24-28).

\section{Results}

Inhibitory effect of Huaier aqueous extract. We used MTT assays to confirm the inhibitory effect of Huaier aqueous extract and to choose the suitable concentration and time of Huaier treatment for microarray assay. The results are shown in Fig. 1. MDA-MB-231 cells were treated with different concentrated solutions and incubated for 24,48 or $72 \mathrm{~h}$ individually. With the higher concentration and longer reaction time, the viability of breast cancer cells was suppressed more

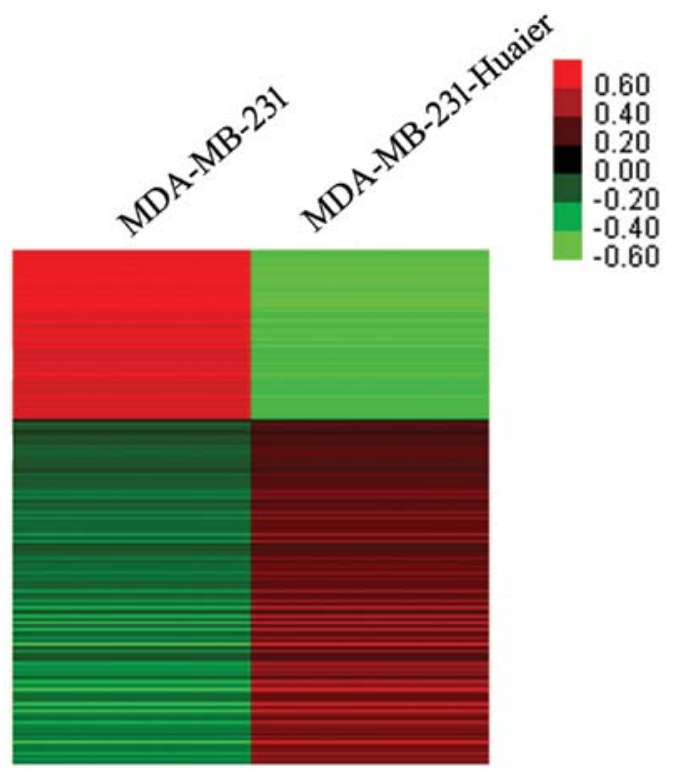

Figure 2. Heat map of differentially expressed genes (DEGs) with expression change fold $>2$. MDA-MB-231 cells were treated by $8 \mathrm{mg} / \mathrm{ml}$ Huaier aqueous extract for $72 \mathrm{~h}$. Rows represent the genes and columns represent the samples. Red blocks represent high and green blocks low expression relative to the control parent cells.

obviously. Huaier aqueous extract showed significant doseand time-dependent effects.

Screening of differentially expressed genes (DEGs). According to the results of MTT assay, we chose the concentration of $8 \mathrm{mg} / \mathrm{ml} \mathrm{Huaier}$ aqueous extract and reaction time of $72 \mathrm{~h}$ to treat MDA-MB-231 cells. After treatment, cells were collected and DEGs were screened by HTA 2.0 Transcriptome Microarray Assay according to methods mentioned above.

After treatment of Huaier aqueous extract, the expression of 387 genes (226 upregulated and 161 downregulated) was changed by at least 2-fold (varying between 2.0-fold and 13.0 -fold) in MDA-MB-231 cells. The top 5 (according to fold change) down- and up-regulated genes in Huaier-treatment cells are shown in Table I. The heat map (Fig. 2) showed DEGs with expression change fold $>2$ from microarray data $(\mathrm{p}<0.05)$. 


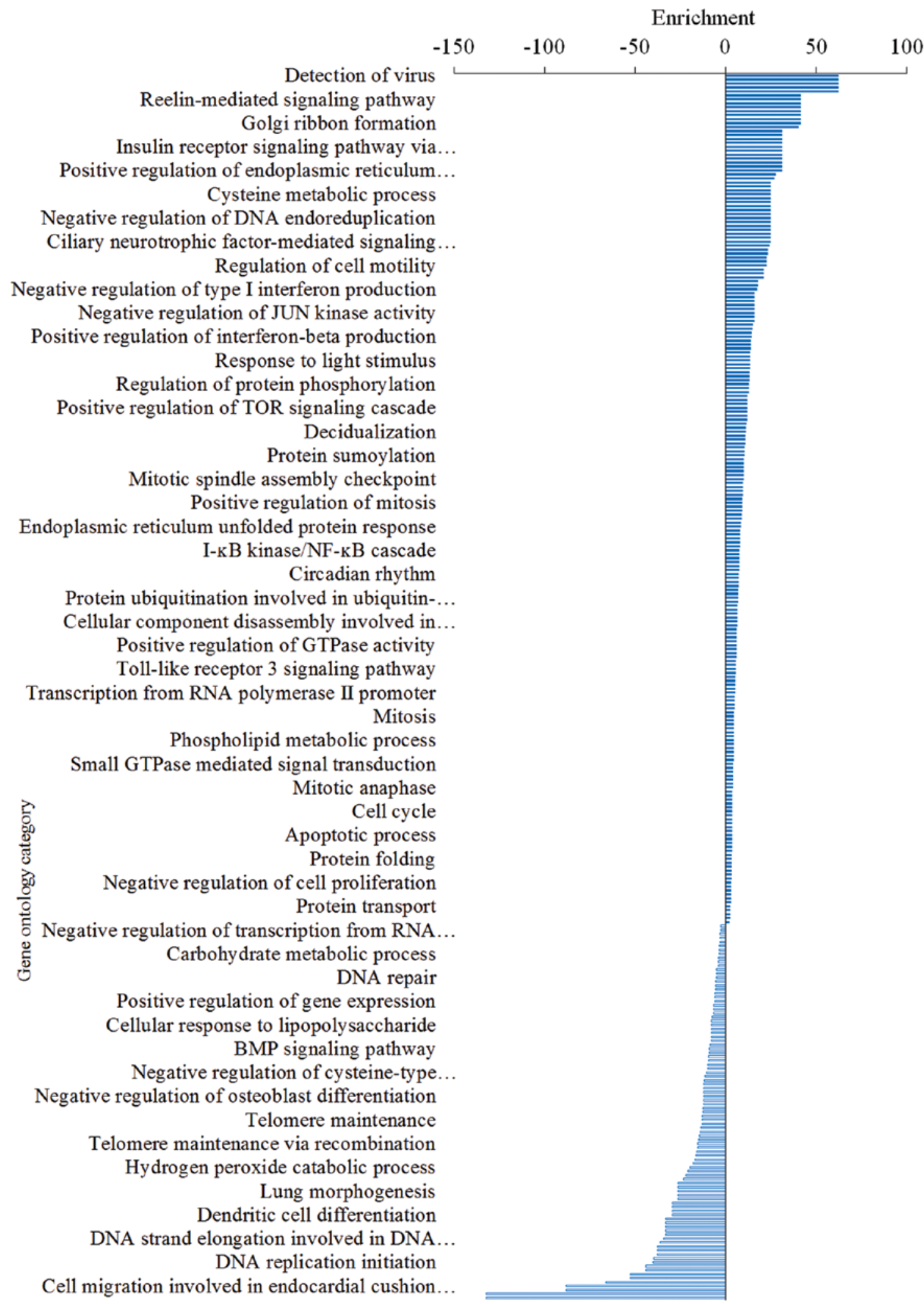

Figure 3. The enrichment of GO categories. Blue solid columns indicate upregulated GO categories and blue hollow columns indicate downregulated GO categories.

Huaier-related biological process. GO and KEGG pathway analysis was applied to identify the altered biological functions of breast cancer cells after treatment of Huaier aqueous extract. Figs. 3 and 4 show the GO analysis results of MDA-MB-231 cells. In addition, Fig. 5 shows the results of KEGG pathway analysis. Larger enrichment value indicated that the function was affected more by Huaier treatment. And larger $-\operatorname{Lg}(\mathrm{p}$-value) indicated that the function was regulated more obviously. Based on these results, treatment with Huaier aqueous extract affected obviously, a number of functions including proliferation, apoptosis, stem cells related functions, autophagy and angiogenesis. 


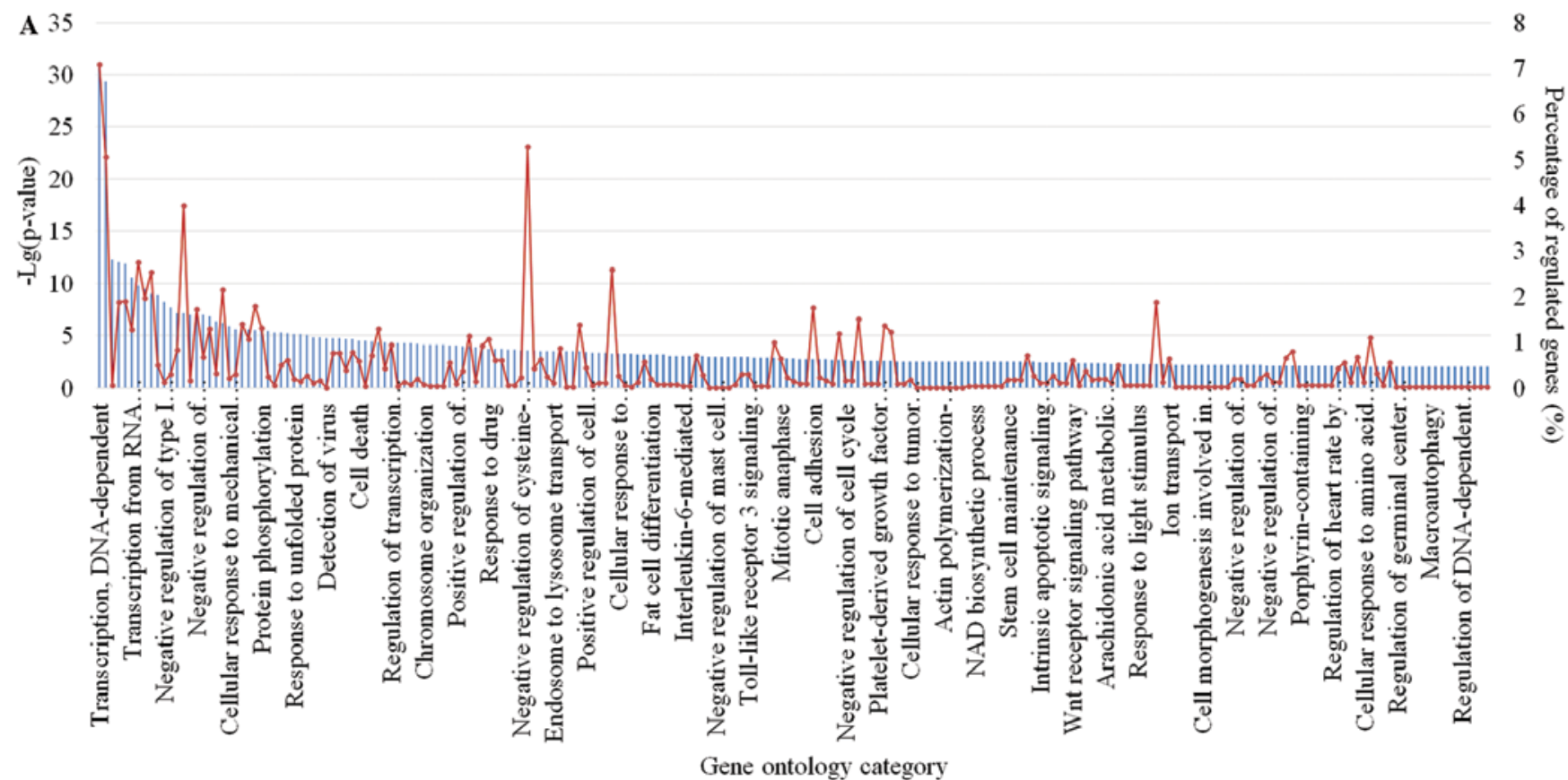

B 18

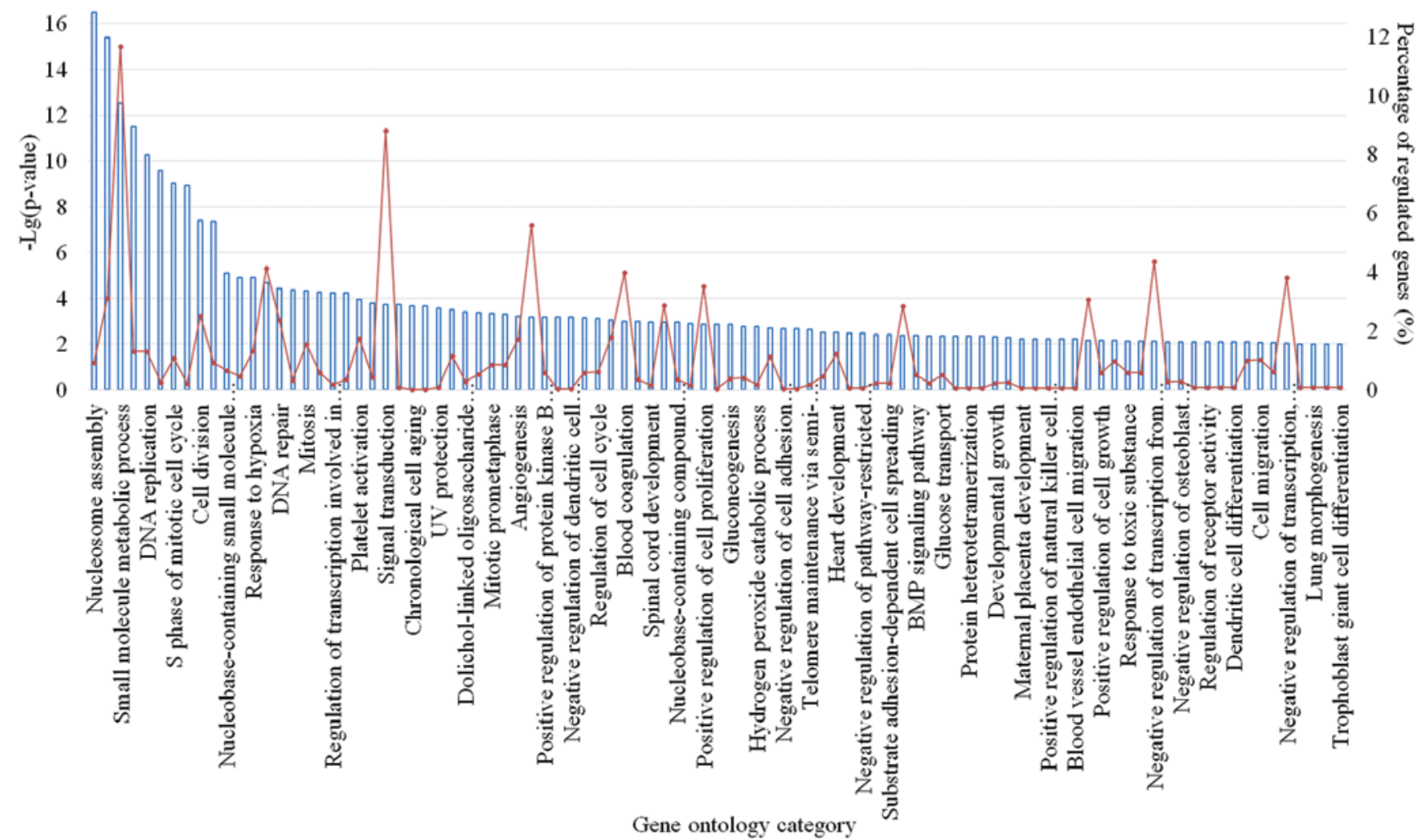

Figure 4. GO analysis results of MDA-MB-231 cells after Huaier treatment. - $\operatorname{Lg}(\mathrm{p}$-value) and percentage of regulated genes of upregulated (A) and downregulated (B) GO categories. Blue solid columns indicate upregulated GO categories and blue hollow columns indicate downregulated GO categories. Red line charts indicate the percentage of regulated genes each category contains.

Gene-gene interaction network. Based on KEGG data base, we could find the relationships between genes, and gene-gene interaction network (Figs. 6 and 7) was constructed. As shown in Fig. 6, these genes of interest were closely connected and most of them were located in the center of the network. To better describe the characteristics of these genes in the network, betweenness centrality and degree were calculated.
Betweenness centrality indicated the intermediary ability of each gene. Larger betweenness centrality value meant greater ability of regulation of genes. Degree represents the number of genes which interacted. Fig. 7 shows the genegene interaction network of angiogenesis and proliferation, apoptosis, and cell cycle, respectively. Table II shows the top 10 important genes in the gene-gene interaction network. 


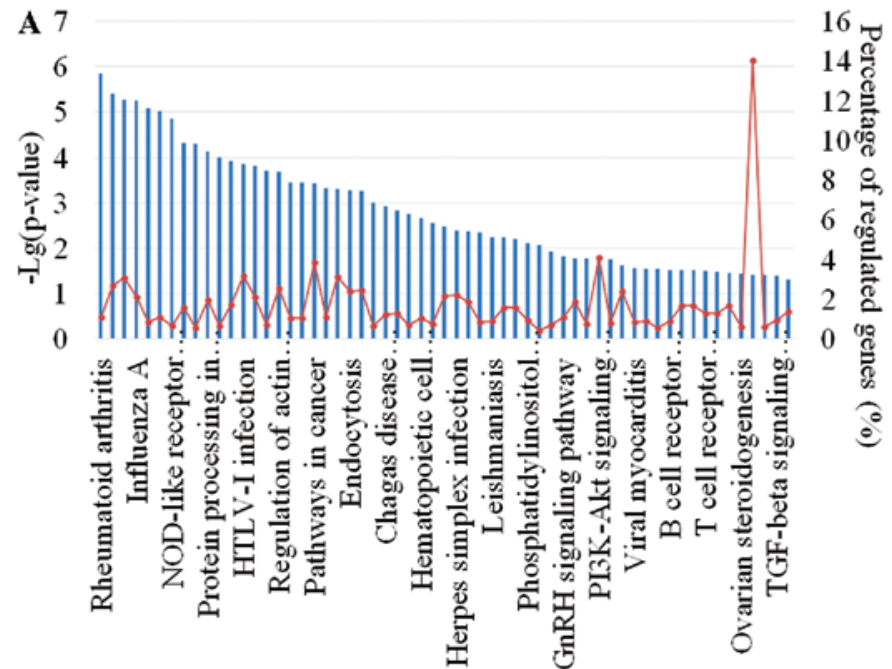

KEGG pathway category

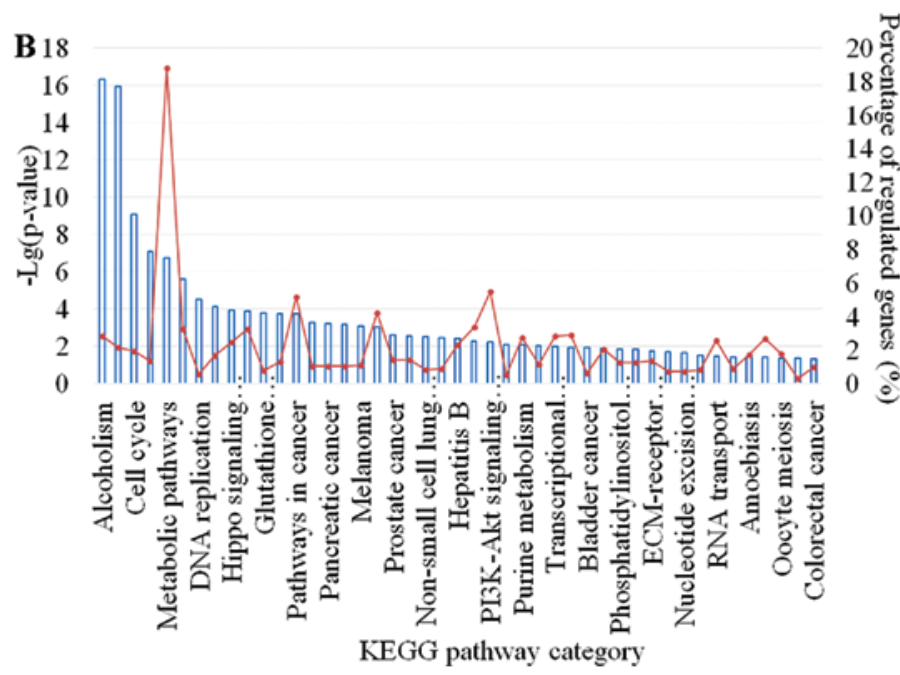

Figure 5. KEGG pathway analysis of MDA-MB-231 cells after Huaier treatment. - $\mathrm{Lg}(\mathrm{p}$-value) and percentage of regulated genes of upregulated (A) and downregulated (B) KEGG pathway categories. Blue solid columns indicate upregulated pathway categories and blue hollow columns indicate downregulated pathway categories. The red line charts indicate the percentage of regulated genes in each category.

Table II. The top 10 important genes in gene-gene interaction network in MDA-MB-231 cells after Huaier treatment.

\begin{tabular}{llccr}
\hline Gene symbol & \multicolumn{1}{c}{ Description } & Regulation style & Betweenness centrality & Degree \\
\hline SQSTM1 & Sequestosome 1 & Up & 0.047686308 & 36 \\
ATM & Ataxia telangiectasia mutated & Up & 0.026535071 & 24 \\
PPP1R12A & Protein phosphatase 1, regulatory subunit 12A & Up & 0.02293258 & 14 \\
SFN & Stratifin & Down & 0.017149581 & 20 \\
PPAP2C & Phosphatidic acid phosphatase type 2C & Down & 0.016731582 & 5 \\
ROCK2 & Rho-associated, coiled-coil containing protein kinase 2 & Up & 0.014117349 & 22 \\
CDK4 & Cyclin-dependent kinase 4 & Down & 0.01203365 & 20 \\
E2F1 & E2F transcription factor 1 & Down & 0.011981357 & 9 \\
APC & Adenomatous polyposis coli & Up & 0.011630106 & 9 \\
PIK3C2A & Phosphoinositide-3-kinase, class 2, $\alpha$ polypeptide & Up & 0.011319546 & 12 \\
\hline
\end{tabular}

\section{Discussion}

Breast cancer is one of the most common malignant tumors in the world. Although the incidence of breast cancer has increased annually, the death rate continues to decline because of early diagnosis and multimodality therapy (1-3). However, still many breast cancer patients inevitably suffer from relapse or metastasis and die from this disease. 


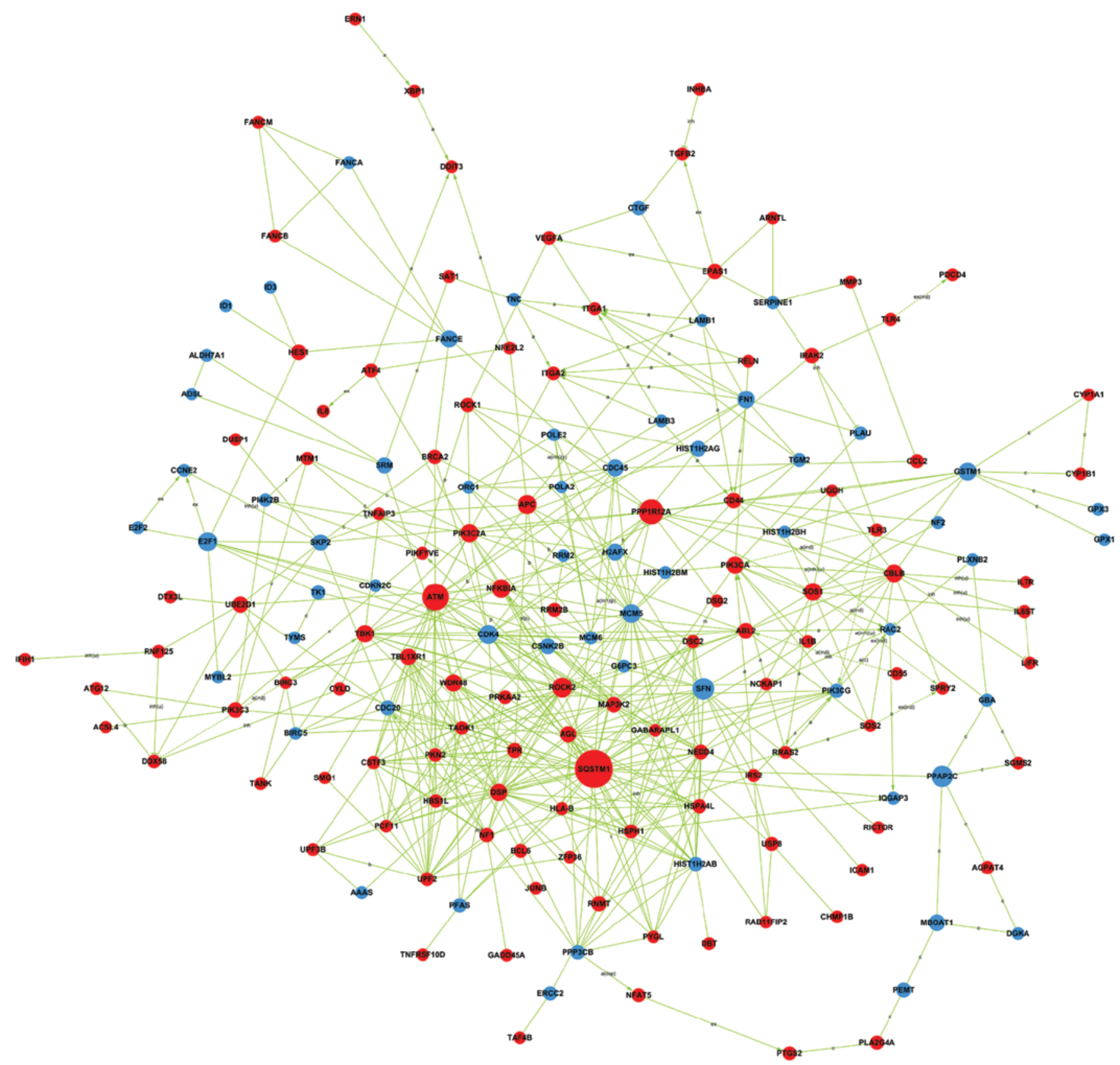

Figure 6. Complete gene-gene interaction network of MDA-MB-231 cells after Huaier treatment. Red indicates upregulated genes and blue indicates downregulated genes. The area of circles indicates the value of betweenness centrality. Line segments indicate gene-gene interaction.

Maintenance treatment is very important for breast cancer patients. For ER positive breast cancer, long-term endocrine therapy is the effective maintenance treatment. Large randomized trials have shown that 10 years of adjuvant endocrine therapy is superior to 5 years (6), showing the value of the long-term maintenance treatment. In comparison, chemotherapy is the only systemic therapy for TNBC. Management of TNBC is a challenge due to lack of targeted therapy, aggressive tumor behavior and relatively poor prognosis (29). Although it has been reported that TNBC was chemosensitive, the results remained unsatisfactory (30-33). Maximal effort should be made to select the best possible drugs for effective maintenance treatment of TNBC.

TCM has a very long history in China. In modern medical research many kinds of TCM have been found with antitumor effects (9). Trametes robiniophila murr (Huaier) is a kind of officinal fungi in China. In recent decades it has been used as a complementary maintenance treatment mostly in liver cancer and breast cancer. However, its mechanisms are still unclear. In recent three or four years, studies on Huaier extract began to appear. The use of Huaier in liver cancer is relatively mature. Until now it has been reported that Huaier extract could inhibit liver cancer from multiple perspectives (34-37). However, there are few studies on the mechanisms of Huaier extract in breast cancer. Therefore, we conducted this study to clarify the possible effects.

Huaier extract consists of many kinds of ingredients. Proteoglycan was confirmed to be the most effective ingredient. However, it was reported that the inhibitory effect of proteoglycan was less effective than the Huaier extract $(15,16)$. 
$\mathbf{A}$
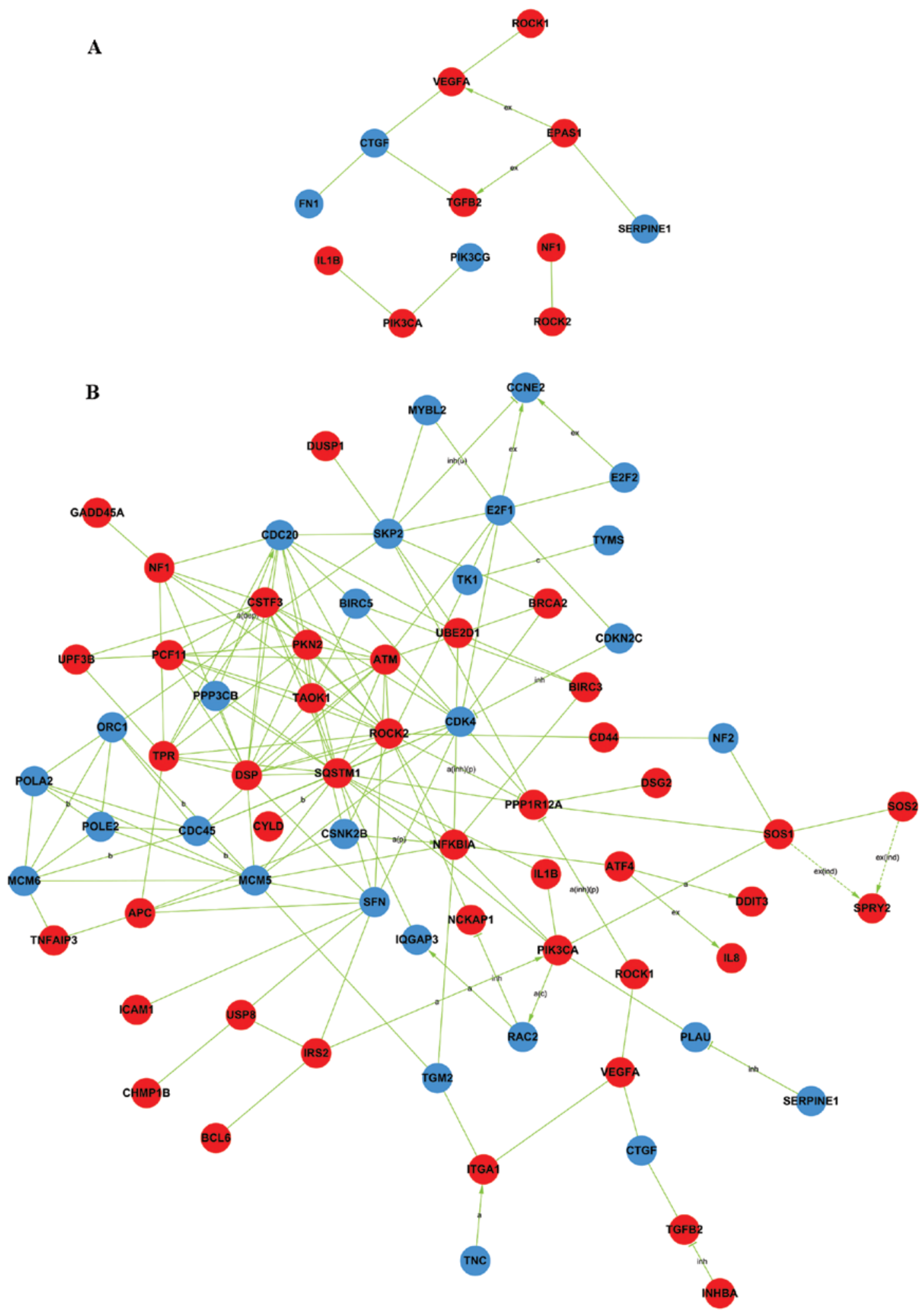

Figure 7. Gene-gene interaction network of MDA-MB-231 cells after Huaier treatment. (A) Gene-gene interaction network on regulation of angiogenesis. (B) Gene-gene interaction network on regulation of proliferation, apoptosis and the cell cycle. Red indicates upregulated genes and blue indicates downregulated genes. The area of the circles indicates the value of betweenness centrality. Line segments indicate gene-gene interaction.

It indicated that the effects of Huaier in the clinical use are not only because of the proteoglycan. In addition Huaier extract was the one which was applied in the clinical work but not proteoglycan. Thus in our study we chose Huaier aqueous extract to treat breast cancer cells and to investigate its mechanisms.
Microarray profiling has given great guidance for modern medical research. We used HTA 2.0 Transcriptome Microarray Assay to analyze the effects of Huaier aqueous extract. MDA-MB-231 cells, which are the most often used in TNBC research, were selected to be treated by Huaier extract. 
Preliminary screening of DEGs gave 387 genes (226 upand 161 down-regulated) in MDA-MB-231 cells which were regulated significantly. Table I lists the top 5 up- and downregulated genes in MDA-MB-231 cells. Due to the limits of DEGs screening, we further conducted the GO analysis, KEGG pathway analysis and gene-gene interaction network analysis to provide a more intuitive result of the effects of Huaier extract.

Figs. 3 and 4 provide the results of GO analysis. Due to the count of genes and degree of enrichments of each GO categories, Fig. 3 was constructed according to the enrichment ranks. In Fig. 3, the GO category with larger enrichment ranked the top and was more affected by Huaier extract when the $p$-values were equal. According to the p-values of each GO category, we constructed Fig. 4, providing the $\mathrm{p}$-values and gene numbers of each category. From the results of GO analysis, we found that many key steps during the breast cancer development and progression were regulated significantly by Huaier extract, including DNA-dependent transcription, apoptotic process, cell cycle arrest, cellular response to hypoxia, immune response, DNA replication, and cell proliferation. It gave us preliminary analysis of the multi-target effects of Huaier extract.

Results of KEGG pathway analysis are shown in Fig. 5. There were 59 pathways containing genes which were upregulated and 45 pathways downregulated. Pathways, such as proteoglycans in cancer, MAPK signaling pathway, NF- $\mathrm{KB}$ signaling pathway, apoptosis, cell cycle, DNA replication and metabolic pathways, were significantly regulated. These results suggested further that Huaier extract inhibited breast cancer from multiple perspectives.

According to the methods described above, we constructed the gene-gene interaction network (Figs. 6 and 7) to fully understand the relationships between the gene groups. Based on these results, some traditional key genes play important roles in the regulation of Huaier extract treatment, including SQSTM1, ATM, CDK4 and E2F1. It provided explicit research fields for future study.

All the analyses described above indicated that many functions and pathways were regulated obviously by Huaier extract. Based on these results, we further studied the effects of Huaier extract in detail. Fig. 7B shows part of the genegene interaction network on proliferation, apoptosis and the cell cycle. According to this result, we found that cell invasion and migration were suppressed with exposure to Huaier extract. Huaier was able to induce G0/G1 cell cycle arrest and p53 accumulation and activation. Breast cancer cell apoptosis executed by caspase-3 were induced by Huaier extract through the mitochondrial pathway (38). In a similar way, Fig. 7A showed the regulated genes which were related with angiogenesis. Based on this, we found that treatment with Huaier extract inhibited angiogenesis in a dose-dependent manner and decreased the levels of phosphorylated extracellular signal-regulated kinase (ERK), transcription factor p65, c-Jun N-terminal kinase (JNK), signal transducer and activator of transcription 3 (STAT3) and the expression of vascular endothelial growth factor (VEGF) (39). Results of microarray also hinted at the regulation of metabolic, immune and stem-like characteristics by Huaier extract, thus, these altered functions will be our future research directions.
In conclusion, microarray profiling showed the multi-target effects of Huaier extract and provided us various research fields. Based on the results of microarray, we further studied the functions of Huaier and confirmed its key roles in treatment of TNBC. All these studies indicated that Huaier extract had multiple effects in breast cancer therapy and could be an important potential maintenance treatment drug for TNBC.

\section{Acknowledgements}

This work was supported by National Natural Science Foundation of China (no. 81172529; no. 81272903) and Shandong Science and Technology Development Plan (no. 2013GRC31801).

\section{References}

1. DeSantis C, Ma J, Bryan L and Jemal A: Breast cancer statistics, 2013. CA Cancer J Clin 64: 52-62, 2014.

2. Chen WQ, Zheng RS, Zhang SW, Zeng HM and Zou XN: The incidences and mortalities of major cancers in China, 2010. Chin J Cancer 33: 402-405, 2014.

3. Siegel R, Naishadham D and Jemal A: Cancer statistics, 2013. CA Cancer J Clin 63: 11-30, 2013.

4. Early Breast Cancer Trialists' Collaborative Group (EBCTCG), Davies C, Godwin J, Gray R, et al: Early Breast Cancer Trialists Collaborative Group (EBCTCG): Relevance of breast cancer hormone receptors and other factors to the efficacy of adjuvant tamoxifen: Patient-level meta-analysis of randomised trials. Lancet 378: 771-784, 2011.

5. Williams $\mathrm{N}$ and Harris LN: The renaissance of endocrine therapy in breast cancer. Curr Opin Obstet Gynecol 26: 41-47, 2014.

6. Davies C, Pan H, Godwin J, et al: Adjuvant Tamoxifen: Longer Against Shorter (ATLAS) Collaborative Group: Long-term effects of continuing adjuvant tamoxifen to 10 years versus stopping at 5 years after diagnosis of oestrogen receptor-positive breast cancer: ATLAS, a randomised trial. Lancet 381: 805-816, 2013.

7. Baum M, Budzar AU, Cuzick J, Forbes J, Houghton JH, Klijn JG and Sahmoud T; ATAC Trialists' Group: Anastrozole alone or in combination with tamoxifen versus tamoxifen alone for adjuvant treatment of postmenopausal women with early breast cancer: First results of the ATAC randomised trial. Lancet 359: 2131-2139, 2002.

8. Giobbie-Hurder A, Price KN, Gelber RD, International Breast Cancer Study Group; BIG 1-98 Collaborative Group: Design, conduct, and analyses of Breast International Group (BIG) 1-98: A randomized, double-blind, phase-III study comparing letrozole and tamoxifen as adjuvant endocrine therapy for postmenopausal women with receptor-positive, early breast cancer. Clin Trials 6: 272-287, 2009.

9. You L, An R, Liang K and Wang X: Anti-breast cancer agents from Chinese herbal medicines. Mini Rev Med Chem 13: 101-105, 2013.

10. Wang J,Liu Q and Yang Q: Radiosensitization effects of berberine on human breast cancer cells. Int J Mol Med 30: 1166-1172, 2012.

11. Franek KJ, Zhou Z, Zhang WD and Chen WY: In vitro studies of baicalin alone or in combination with Salvia miltiorrhiza extract as a potential anti-cancer agent. Int J Oncol 26: 217-224, 2005.

12. Pozo-GuisadoE,Merino JM,Mulero-Navarro S, et al: Resveratrolinduced apoptosis in MCF-7 human breast cancer cells involves a caspase-independent mechanism with downregulation of Bcl-2 and NF-kappaB. Int J Cancer 115: 74-84, 2005.

13. Wu C, Chen F, Rushing JW, Wang X, Kim HJ, Huang G, HaleyZitlin V and He G: Antiproliferative activities of parthenolide and golden feverfew extract against three human cancer cell lines. J Med Food 9: 55-61, 2006.

14. Li L, Ye S, Wang Y and Tang Z: Progress on experimental research and clinical application of Trametes robiniophila. China Cancer 16: 110-113, 2006.

15. Guo Y, Cheng P, Chen Y, et al: Studies on the constituents of polysaccharide from the hyphae of trametes robiniophila (II) identification of polysaccharide from the hyphae of trametes robiniophila and determination of its molar ratio. J China Pharm Univ 23: 155-157, 1992 (In Chinese). 
16. Guo Y, Cheng P, Chen Y, et al: Isolation and analysis of the polysaccharide of Huaier mycelium. Chin J Biochem Pharm 63: 56-59, 1993.

17. Gene Ontology Consortium: The Gene Ontology (GO) project in 2006. Nucleic Acids Res 34: D322-D326, 2006.

18. Ashburner M, Ball CA, Blake JA, et al; The Gene Ontology Consortium: Gene ontology: Tool for the unification of biology. Nat Genet 25: 25-29, 2000.

19. Dupuy D, Bertin N, Hidalgo CA, et al: Genome-scale analysis of in vivo spatiotemporal promoter activity in Caenorhabditis elegans. Nat Biotechnol 25: 663-668, 2007.

20. Schlitt T, Palin K, Rung J, Dietmann S, Lappe M, Ukkonen E and Brazma A: From gene networks to gene function. Genome Res 13: 2568-2576, 2003.

21. Kanehisa M, Goto S, Kawashima S, Okuno Y and Hattori M The KEGG resource for deciphering the genome. Nucleic Acids Res 32: D277-D280, 2004.

22. Yi M, Horton JD, Cohen JC, Hobbs HH and Stephens RM: WholePathwayScope: A comprehensive pathway-based analysis tool for high-throughput data. BMC Bioinformatics 7: 30, 2006.

23. Draghici S, Khatri P, Tarca AL, Amin K, Done A, Voichita C, Georgescu C and Romero R: A systems biology approach for pathway level analysis. Genome Res 17: 1537-1545, 2007.

24. Jansen R, Greenbaum D and Gerstein M: Relating whole-genome expression data with protein-protein interactions. Genome Res 12: 37-46, 2002.

25. $\mathrm{Li} \mathrm{C}$ and $\mathrm{Li} \mathrm{H}$ : Network-constrained regularization and variable selection for analysis of genomic data. Bioinformatics 24 $1175-1182,2008$

26. Wei $\mathrm{Z}$ and Li H: A Markov random field model for network-based analysis of genomic data. Bioinformatics 23: 1537-1544, 2007.

27. Zhang JD and Wiemann S: KEGGgraph: A graph approach to KEGG PATHWAY in R and bioconductor. Bioinformatics 25 1470-1471, 2009.

28. Spirin V and Mirny LA: Protein complexes and functional modules in molecular networks. Proc Natl Acad Sci USA 100: 12123-12128, 2003.
29. Yadav BS, Sharma SC, Chanana P and Jhamb S: Systemic treatment strategies for triple-negative breast cancer. World J Clin Oncol 5: 125-133, 2014.

30. Hernandez-Aya LF, Chavez-Macgregor M, Lei X, et al: Nodal status and clinical outcomes in a large cohort of patients with triple-negative breast cancer. J Clin Oncol 29: 2628-2634, 2011.

31. De Laurentiis M, Cancello G, D'Agostino D, et al: Taxane-based combinations as adjuvant chemotherapy of early breast cancer: A meta-analysis of randomized trials. J Clin Oncol 26: 44-53, 2008.

32. Carey LA, Dees EC, Sawyer L, et al: The triple negative paradox: primary tumor chemosensitivity of breast cancer subtypes. Clin Cancer Res 13: 2329-2334, 2007.

33. Liedtke C, Mazouni C, Hess KR, et al: Response to neoadjuvant therapy and long-term survival in patients with triple-negative breast cancer. J Clin Oncol 26: 1275-1281, 2008.

34. Ren J, Zheng C, Feng G, et al: Inhibitory effect of extract of fungi of Huaier on hepatocellular carcinoma cells. J Huazhong Univ Sci Technolog Med Sci 29: 198-201, 2009.

35. Zheng J, Li C, Wu X, et al: Huaier polysaccharides suppresses hepatocarcinoma MHCC 97-H cell metastasis via inactivation of EMT and AEG-1 pathway. Int J Biol Macromol 64: 106-110, 2014.

36. Li C, Wu X, Zhang H, et al: A Huaier polysaccharide inhibits hepatocellular carcinoma growth and metastasis. Tumour Biol: Nov 6, 2014 (Epub ahead of print).

37. Zou Y, Xiong H, Xiong $\mathrm{H}$, et al: A polysaccharide from mushroom Huaier retards human hepatocellular carcinoma growth, angiogenesis, and metastasis in nude mice. Tumour Biol: Dec 11, 2014 (Epub ahead of print).

38. Zhang N, Kong X, Yan S, Yuan C and Yang Q: Huaier aqueous extract inhibits proliferation of breast cancer cells by inducing apoptosis. Cancer Sci 101: 2375-2383, 2010.

39. Wang X, Zhang N, Huo Q and Yang Q: Anti-angiogenic and antitumor activities of Huaier aqueous extract. Oncol Rep 28 1167-1175, 2012. 